\title{
The rise of colloidal quantum dots
}

\author{
This issue features a theme on colloidal quantum dots, bringing together primary research findings and \\ overviews, along with articles on the commercialization of this technology.
}

It is a commonly held belief that a material's composition defines its optoelectronic properties. Although this is indeed often the case, when semiconductors are scaled down in size, they can behave very differently from their bulk counterparts. Indeed, quantum dots - semiconductor nanocrystals with diameters in the range $2-10 \mathrm{~nm}$ - show size-dependent optical properties due to the effect of quantum confinement. Perhaps the most striking of these properties is that the monochromatic light emission from quantum dots is not only highly efficient but can be tuned by simply changing their diameter.

Furthermore, suspending such quantum dots in an organic polymer solution allows them to be printed and poured like a photonic ink onto a substrate, opening an avenue to easy, low-cost fabrication. Combined with efficient, narrowband light emission, this means that these tiny suspended semiconductor dots are now being explored for cheap, bright LEDs in lighting and displays, fluorescent labels in biomedical imaging and broadband solar cells, to mention just a few applications.

\section{There are currently an}

\section{impressive number of companies commercializing quantum dots and dot-based devices.}

The June issue of Nature Photonics has a special theme on colloidal quantum dots, presenting the latest results from several research groups and commercial companies in this area. This theme gathers a collection of two primary research papers on a quantum dot LED display and an image sensor, a progress article on quantum dot photovoltaics, a News and Views article and an Out of the Lab piece charting commercialization of the technology. The compilation clearly demonstrates the great progress that colloidal quantum dots are making, not only in terms of device performance but also towards deployment in a wide variety of applications.

The LED is one of the devices in which quantum dots can be put into good use thanks to their ease of colour tunability, high brightness and narrow emission bandwidth. Nevertheless, large energy barriers for hole

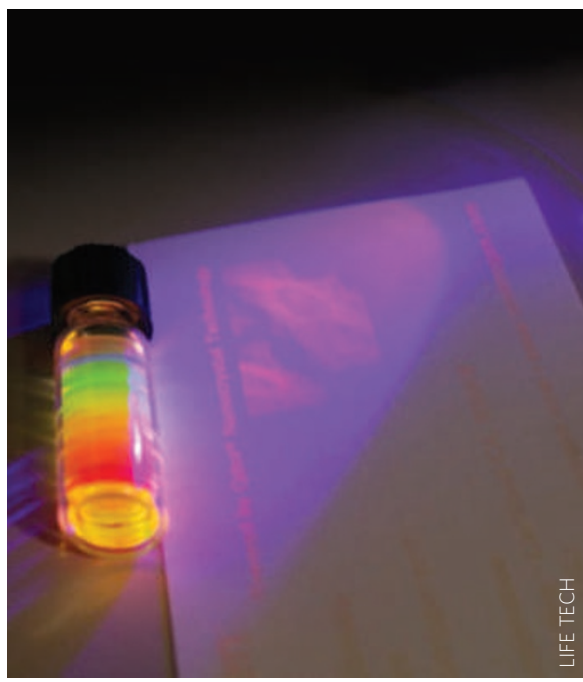

What's interesting about the achievements of these two groups is that they demonstrate that integration of colloidal quantum dot optoelectronics with silicon backplane electronics is practical, which is of great importance if the technology is going to find its way into products.

The findings are put into context by Seth Coe-Sullivan from the quantum dot start-up firm QD Vision in a News and Views article on page 315 , where he shares his thoughts on the role played by colloidal quantum dots in vision and sensing in particular, and on the challenges that remain ${ }^{3}$.

Indeed, a look at the Out of the Lab article on page 307 immediately tells us that colloidal quantum dot technology has already garnered great attention in the world of commercialization ${ }^{4}$. The race to bring quantum dots to the market in fact started back in the early 2000s, and there are currently an impressive number of companies commercializing quantum dots and dot-based devices.

Firms are now able to mass-produce quantum dots with accurate dimensions and a variety of coatings, and are busy exploring their potential in applications ranging from displays and biomedical imaging to photovoltaics and quantum inks for fighting currency counterfeiting.

The prospects for solution-processed photovoltaics based on colloidal quantum dots are outlined in this month's Progress Article by Edward Sargent from the University of Toronto ${ }^{5}$. As he explains on page 325 , the attraction of the technology is that it promises to reduce the cost per area of solar cells and extend their absorption wavelength to infrared region where half of the solar energy lies.

One thing is for sure: the research behind colloidal quantum dots is growing and sooner rather than later will be evident in the world around us in the form of brighter, more colourful displays, energy-efficient lighting and custom-designed fluorescent tags for biological imaging.

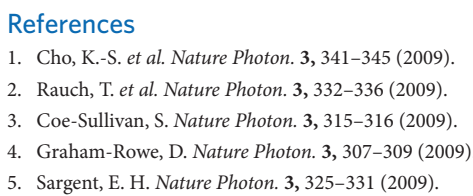

